\title{
UNIT WITT VECTORS
}

\section{MARVIN J. GREENBERG}

1. The multiplicative group $W_{n}^{*}$ of units, in the ring $W_{n}$ of Witt vectors [2] of length $n$ with coefficients in an algebraically closed field $k$ of characteristic $p>0$, is an algebraic group variety over $k$. It decomposes into the direct product of group varieties $W_{n}^{*}=W_{1}^{*}$ $\times U_{n-1}$, where $W_{1}^{*}$ is isomorphic to the one-dimensional multiplicative group $G_{m}$, and $U_{n-1}$ is the algebraic subgroup defined by $x_{0}=1$; the decomposition is given by $\left(x_{0}, \quad\left(1, x_{1}, \ldots, x_{n-1}\right)\right)$ $\rightarrow\left(x_{0}, x_{0}^{p} x_{1}, \cdots, x_{0}^{p^{n-1}} x_{n-1}\right)$. Dieudonné has remarked [1] that $U_{n-1}$ is isomorphic as a formal Lie group to the additive group $W_{n-1}^{+}$. We will show that these groups are isomorphic as algebraic groups only when $p \geqq 3$, and we will determine the structure of $U_{n-1}$ in characteristic 2 .

2. For the analytic assertions in this section, we refer to Hasse $[2, \S 17]$. The Witt vectors of infinite length form a complete discrete valuation ring $W_{\infty}$ of characteristic 0 , with residue field $k$, and maximal ideal generated by the prime $p$. Let

$$
U^{(n)}=\left\{z \in W_{\infty} ; z \equiv 1 \bmod p^{n}\right\}
$$

so that $U^{(1)} / U^{(n+1)}=U_{n}$. Let $U_{n}^{(i)}$ be the subgroup $U^{(i)} / U^{(n+1)}$ of $U_{n}$. The series

$$
\log (1+x)=x-\frac{x^{2}}{2}+\frac{x^{3}}{3}-\cdots+\frac{(-1)^{n-1} x^{n}}{n}+\cdots
$$

converges for all $(1+x) \in U^{(1)}$, and defines an analytic homomorphism from $U^{(n)}$ into the additive group $p^{n} W_{\infty}$ for all $n>0$. Hence it induces by passage to the quotient a homomorphism

$$
\lambda_{n}: U_{n} \rightarrow p W_{\infty} / p^{n+1} W_{\infty} \cong W_{n}^{+} .
$$

$\lambda_{n}$ is a homomorphism of algebraic groups, i.e., is an everywhere defined rational mapping, since only a finite number of terms in the log series contribute to $\lambda_{n}$. We also have the usual exponential series $\exp x$, which converges for all $x$ of order $>1 /(p-1)$, and which defines an analytic homomorphism of $p^{n} W_{\infty}$ into $U^{(n)}$ inverse to the logarithm, provided $n>1 /(p-1)$. Passage to the quotient yields a rational homomorphism

$$
\epsilon_{n}^{(i)}: p^{i} W_{\infty} / p^{n+1} W_{\infty} \rightarrow U_{n}^{(i)}
$$

Received by the editors February 1, 1961. 
inverse to $\lambda_{n} \mid U_{n}^{(i)}$ for all $i>1 /(p-1)$. If $p \geqq 3$, the inequality $i>1 /(p-1)$ holds for all $i>0$. Thus $\lambda_{n}$ is an isomorphism of the algebraic group $U_{n}$ with $W_{n}^{+}$, whose inverse is $\epsilon_{n}^{(1)}$, if the characteristic of $k$ is $\geqq 3$.

3. Assume now $p=2$. Then the restriction of $\lambda_{n}$ to the subgroup $U_{n}^{(2)}$ of $U_{n}$ defined by $x_{1}=0$ is an isomorphism. We have the commutative diagram

$$
\begin{array}{r}
1 \longrightarrow U_{n}^{(2)} \longrightarrow U_{n} \stackrel{\pi}{\longrightarrow} G_{a} \longrightarrow 0 \\
\cong \downarrow \quad \lambda_{n} \downarrow \quad \downarrow \psi \\
0 \longrightarrow W_{n-1}^{+} \stackrel{V}{\longrightarrow} W_{n}^{+} \stackrel{R^{n-1}}{\longrightarrow} G_{a} \longrightarrow 0
\end{array}
$$

where $\pi\left(1, x_{1}, \cdots, x_{n}\right)=x_{1}, G_{a}=W_{1}^{+}$is the one-dimensional additive group, and $V, R$ denote the shift and restriction homomorphisms of Witt vectors. It is clear without introducing $\lambda_{1}$ that $U_{1}$ is isomorphic to $G_{a}$. For $n \geqq 2$, the class of $U_{n}$ in $\operatorname{Ext}\left(G_{a}, W_{n-1}^{+}\right)$will be completely determined by the rational endomorphism $\psi$ of $G_{a}$ (Serre [3, Chapter VII]). Putting $\lambda_{n}\left(1, x_{1}, \cdots, x_{n}\right)=\left(0, y_{1}, \cdots, y_{n}\right)$, we have $\psi\left(x_{1}\right)$ $=y_{1}$. The first two terms in the log series are the only ones which can have order 1 , and a computation of $x-x^{2} / 2$ within $W_{\infty}$ shows that $y_{1}=x_{1}^{2}+x_{1} \cdot \lambda_{n}$ is a separable isogeny of degree 2 whose kernel is $\{ \pm 1\}$. The special property of the prime 2 here seems to be that the 2 -adic logarithm of -1 is well-defined.

Note added in proof. J.-P. Serre has determined the structure of the pro-algebraic group $U^{(1)}$, using a different technique. Cf. his Sur les corps locaux à corps résiduel algébriquement clos, Bull. Soc. Math. France 89 (1961), §1.8, p. 115.

\section{BIBLIOGRAPHY}

1. J. Dieudonné, Lie groups and Lie hyperalgebras over a field of characteristic p>0. II, Amer. J. Math. 77 (1955), 218-244.

2. H. Hasse, Zahlentheorie, Akademie-Verlag, Berlin, 1949.

3. J.-P. Serre, Groupes algebriques et corps de classes, Hermann, Paris, 1959.

University of California, Berkeley 